\title{
Begeisternder Dreiklang für Geist, Ohren und Gaumen
}

\author{
3. trinationaler Phytotherapie-Kongress vom 2.-4. Juni 2016 in Bonn
}

Zum dritten trinationalen Kongress der deutschen, österreichischen und schweizerischen Gesellschaften für Phytotherapie, der vom 2.-4. Juni 2016 zum ersten Mal in Deutschland stattfand, konnten Kongresspräsidentin PD Dr. G. Ulrich-Merzenich (Universitätsklinikum Bonn) und GPT-Präsidentin Prof. K. Kraft mehr als 200 Teilnehmer in der Beethovenstadt Bonn begrüßen. Das Forum Süd der Beethovenhalle bot den teilnehmenden Medizinern, Pharmazeuten, Apothekern und Naturwissenschaftlern ein angenehmes Ambiente zu Vorträgen und Diskussionen über die Zukunft der Phytotherapie.

Dass der Kongressort zum Beethovenjubiläum im Jahr 2020 grundlegend modernisiert und zukunftsfähig gestaltet wird, kann im übertragenen Sinne auch für die Phytotherapie gelten: Sie hat derzeit durch den Einsatz modernster Forschungsmethoden aus Molekularbiologie und Chemie die Chance zu einer rasanten und vielseitigen Weiterentwicklung. In diesem Sinne begrüßten Bürgermeisterin G. Klingmüller genauso wie Prof. N. Wernert, Dekan der Medizinischen Fakultät, sowie Prof. G. König, Institutsleiterin der Pharmazeutischen Biologie, und Prof. A.R. Bilia, Präsidentin der GA, die Teilnehmer mit ihrem Esprit und wünschten dem Kongress einen erfolgreichen Verlauf.

\section{Forschung und Entwicklung brauchen Mut}

In seiner Eröffnungsrede kritisierte Prof. A. Hensel zwar zunächst die Stagnation der Forschung und Entwicklung (F\&E),

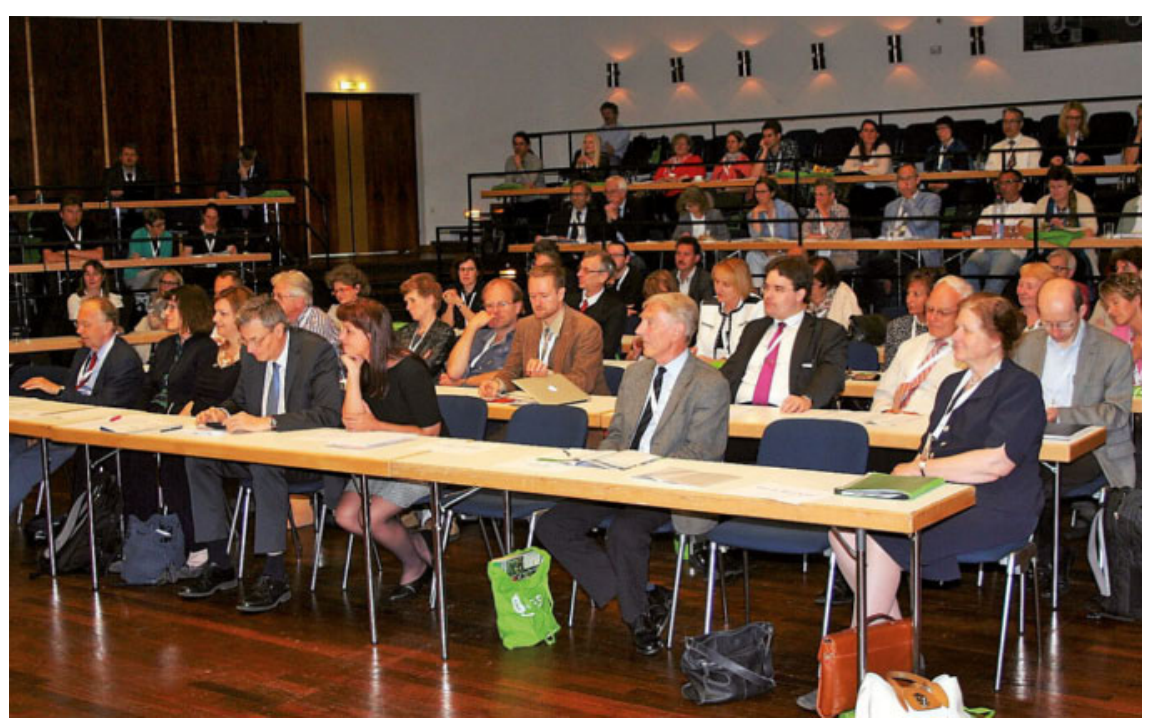

Drei Tage konzentrierte Phytotherapie: Der trinationale Kongress 2016 in der Bonner Beethovenhalle.

die er für die gesamte (phyto)-pharmazeutische Branche beobachtet, aber er berichtete auch über Mut machende gelungene Beispiele von Neuzulassungen, z.B. des Procyanidin-angereicherten Extrakts aus Croton lechleri zur Behandlung der HIV/AIDS assoziierten Diarrhö. Gleichzeitig zeigte er auf, dass Phytopharmaka innovative Wirkmechanismen für Neuentwicklungen bereithalten und schlug mutige und kreative Finanzierungskonzepte für F\&E mit dem Ziel von Arzneimittelinnovationen auf der Basis von Naturstoffen vor.

Dr. M. Rostock, Onkologe am Universitäts-Spital Zürich und dem universitären Krebszentrum Hamburg Eppendorf, berichtete von bereits erfolgreich praktizierten „Innovationen“ bei der unterstützenden Behandlung des Mamma-Karzinoms, z.B. den positiven Studienergebnissen für Ginsengwurzel-, Guarana- und auch Baldrianwurzelextrakte bei ausgeprägter tumorassoziierter Fatigue sowie den phytotherapeutischen Begleittherapien zur Verbesserung der Lebensqualität unter Chemotherapie. Er betonte allerdings, dass bei Begleittherapien auch mögliche Risiken unerwünschter Effekte bedacht werden müssen und dass zur Frage der Verlängerung der Lebenszeit aufgrund mangelnder Daten bisher keine gesicherten Aussagen möglich sind.

Dr. O. Kelber zeigte die erfolgreiche Anwendung von Phytopharmaka an Beispielen aus der Gastroenterologie (Reizdarmsyndrom, Colitis ulcerosa) und der Depression auf. Bei diesen Indikationen ist die Phytotherapie bereits mit positiven Aussagen in den ärztlichen Leitlinien erwähnt und für Kreuzschmerz sogar in die Nationalen Versorgungsleitlinien aufgenommen worden. 


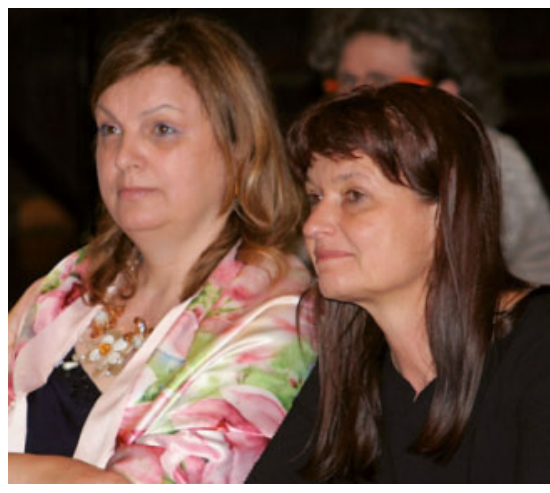

Prof. Anna Rita Bilia, Präsidentin der GA, und PD Dr. G. Ulrich-Merzenich, Kongresspräsidentin.

\section{Ehrensymposium für Heinz Schilcher}

Die dem verstorbenen Prof. Heinz Schilcher gewidmete zweite Session des Kongresses spiegelte die herausragende Rolle von Prof. Schilcher wider, der maßgeblich den Weg für die Standardisierung der Phytopharmaka seit Anfang der 60er-Jahre mitgestaltete. Sein Wirkungsfeld und Leben auch außerhalb der Pharmazie, insbesondere im Sport als Deutscher Kanu-Meister sowie als Teamleiter während der Olympischen Spiele 1972 wurden gewürdigt und von seinem Freund Dr. B. Frank mit fotografischen Erinnerungen wachgerufen. Die folgenden Beiträge von

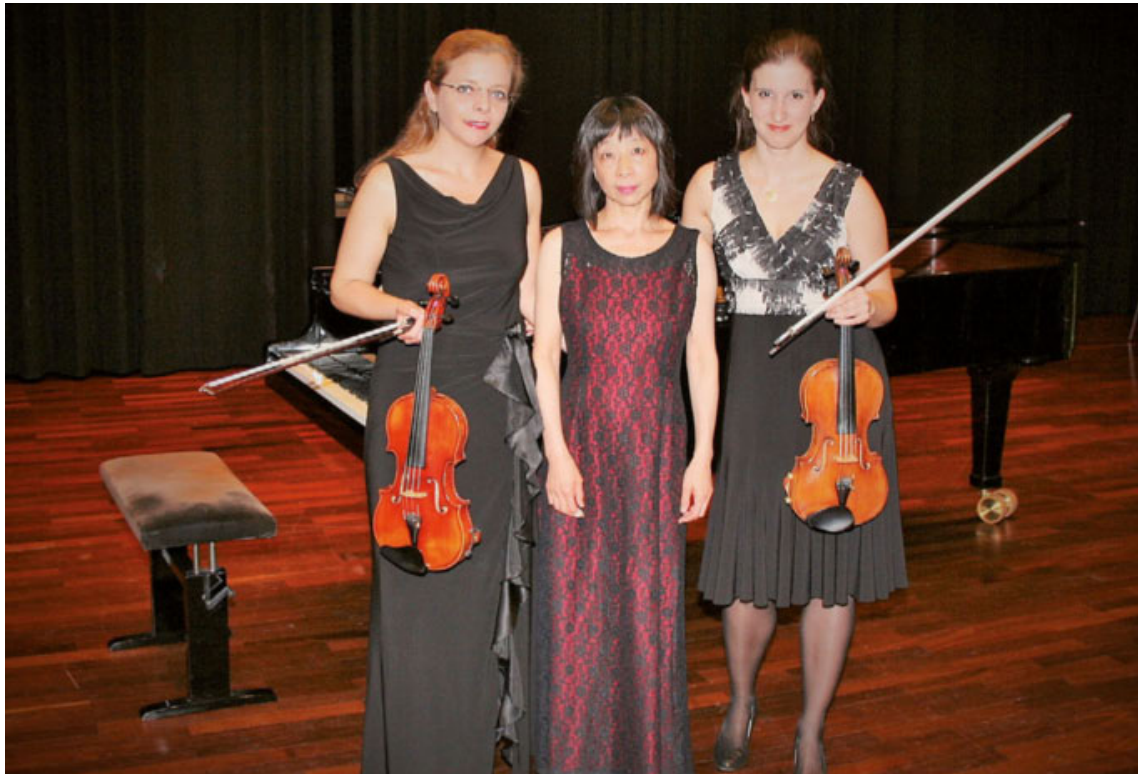

Ein besonderer Genuss: Das Kammerkonzert mit Sonja Wiedebusch, Eriko Yamamoto und Susanne Rohe. pflanzen ausreichend untersucht sind und hier für die phytotherapeutische Forschung mehr Geld zur Verfügung gestellt werden sollte, um ihrem tatsächlichen Stellenwert in der Gesellschaft gerecht zu werden.

\section{Ein Forum für junge Wissenschaftler}

Ein spezielles Treffen bot jungen Wissenschaftlern die Gelegenheit, ihre ersten wissenschaftlichen oder therapeutischen Erfahrungen in der Beschäftigung mit Phytomedizin vorzustellen und auszutauschen. Die Phytotherapie-Kongresse wollen zukünftig einen besonderen Akzent darauf legen, junge Lebenswissenschaftler für die Forschung mit pflanzlichen Heilmitteln zu begeistern.

Für die Posterbesichtigung wie auch für die Industrieausstellung wurden angemessene Zeitfenster eingerichtet, um diese Beiträge im Rahmen des Kongresses zu würdigen. Hier wurden aktuelle Forschungsergebnisse und neue Projekte vorgestellt. Auch Arbeiten zu nichteuropäischen Arzneipflanzen, z.B. komplexe Rezepturen aus der Tibetischen Medizin, aus der japanischen Kampo-Medizin sowie Arzneipflanzen aus der Karibik, die in die europäische Phytotherapie integriert werden sollen, wurden diskutiert. Die Ausstellung eines Zyklus von Arzneipflanzenbildern der Künstlerin Violetta Vollrath rundete die Posterpräsentation in kreativer Weise ab.

\section{Beethoven am Abend}

Den gelungenen Abschluss des ersten Kongresstages bildete ein Kammerkonzert mit den Musikerinnen Sonja Wiedebusch (Violine) und Susanne Rohe (Violine) vom Beethoven-Orchester sowie Eriko Yamamto (Klavier) im „Studio“. Das Ensemble verzauberte die Zuhörer auf hohem Kunst- und Genussniveau mit Klängen von Bach, Beethoven, Vivaldi und Schostakowitsch. Die Zuhörer dankten mit begeisterten standing Ovations und gingen mit viel positiver Energie in den nächsten Tag des Kongresses. 


\section{Leitlinienarbeit und}

\section{Versorgungsforschung}

In der Session zur Versorgungsforschung zu Beginn des zweiten Kongresstages stellte Prof. K. Kraft in Vertretung von Prof. J. Langhorst dar, dass Phytotherapeutika mittlerweile in 40 ärztlichen Leitlinien und auch in eine Nationale Versorgungsleitlinie eingebracht werden konnten. Möglich wurde dies nicht zuletzt dadurch, dass die GPT Ende 2013 Mitglied in der Arbeitsgemeinschaft der Wissenschaftlichen Medizinischen Fachgesellschaften (AWMF) wurde und somit einen aktiven Fachvertreter in die Kommissionen zur Erstellung der Leitlinien entsendet. Prof. W. Lehmacher brachte anschließend den Zuhörern sehr anschaulich und kurzweilig näher, wie versorgungsrelevante Vergleichsstudien zur Verbesserung der Evidenz von Phytopharmaka führen können - mit den Hinweis, dass sich neben der Industrie auch öffentliche Förderstellen, Stiftungen und Wissenschaftsorganisationen an der Förderung derartiger Studien beteiligen sollten, gerade auch wegen des hohen allgemeinen Interesses an Phytotherapeutika. Dr. G. Meng stellte schließlich die wissenschaftliche Datenbank „PhytoVIS“ der Kooperation Phytopharmaka vor, die als Plattform für epidemiologische Studien zur Versorgungsforschung mit pflanzlichen Arzneimitteln dient und bereits ca. 20000 Patientendatensätze erfasst hat.

\section{Synergieforschung}

Auch die Synergieforschung und die „Omics“-Technologien durften auf dem Kongress nicht fehlen, sind es doch diese Methoden, deren Einsatz die Grundlagenforschung zu Phytotherapeutika massiv stärkt und bereits jetzt und noch sehr viel mehr in der Zukunft wesentliche Beiträge dazu liefern wird, die komplexen Wirkungsmechanismen von Arzneipflanzen aufzuklären. PD Dr. G. Ulrich-Merzenich gab einen anschaulichen Überblick über die Entwicklung und den derzeitigen Stand der Synergieforschung, die für manchen Zuhörer aufgrund der unvermeidbaren Exkurse in mathematische Grundlagen vielleicht manchmal etwas „ungewohnte Kost“ darstellte, aber gleichzeitig andeutete, welch großes Potenzial eine zukünftige personalisierte Medizin auf der Basis synergistischer Wirkungen gerade auch für pflanzliche Wirkstoffe bietet. Prof. W. Dimpfel zeigte anschließend auf, wie anhand der Analyse von Hirnströmen sogenannte Elektropharmakogramme von pflanzlichen Arzneimitteln erstellt werden können. Diese scheinen auch Schlussfolgerungen auf die Wirkungsweise von pflanzlichen Mischextrakten auf das menschliche Gehirn zu erlauben. Prof. A. Hensel stellte in einem spannenden Vortrag neuartige sulfatierte Flavonglucuronide und Scopoletinglykoside aus den Wurzeln von Althaea officinalis L. mit ihren pharmakologischen Wirkungen auf Extrazellulärmatrixmoleküle vor. Dr. H. Schwabl legte anhand von tibetischen Rezepturen nochmals dar, dass nicht die Summe der einzelnen Inhaltsstoffe allein die Wirkung charakterisiert. Multikomponent-Mischungen wie Arzneipflanzenextrakte besitzen pleiotrope Signaturen und können als Prototypen der „herbal“ Network Pharmacology untersucht werden.

\section{Mit Diskussionsbedarf: Regulatorische Rahmen- bedingungen}

Der Nachmittag des zweiten Kongresstages wurde gestaltet durch 4 Parallelsessions mit den Schwerpunkten „Regulatorische Rahmenbedingungen, Indikationen, Novel Targets und Extraktentwicklung“.

In der regulatorische Session standen Prof. W. Knöss (D), Dr. R. Länger (A) und Dr. M. Ziak (CH) dem Publikum Rede und Antwort bezüglich der Zulassungen von Phytotherapeutika im deutschsprachigen Raum. Die anfänglichen Fragen zur Zulassung von Kombinationspräparaten weiteten sich schnell aus zu Fragen bezüglich der Rolle von Tierversuchen in der phytotherapeutische Forschung. Es kam zu regen Diskussionen zwischen Publikum und Vertretern der Behörden, moderiert von Dr. H. Pittner. Diese Session machte deutlich, dass der trinationale Kongress eine ideale Plattform für rege Diskussio-

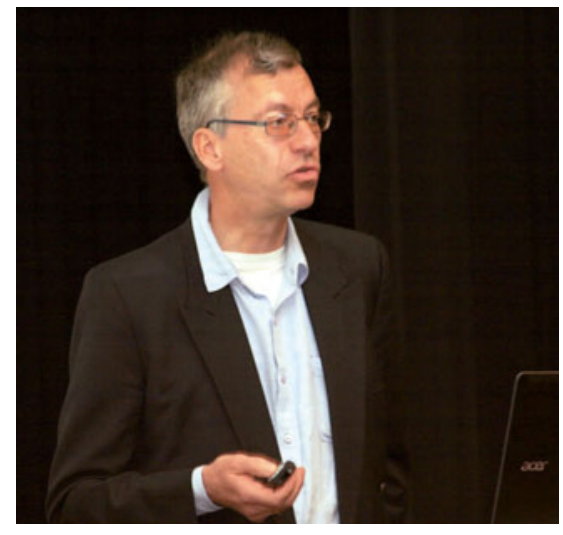

Prof. Dr. Andreas Hensel.

nen und Absprachen über die regulatorischen Rahmenbedingungen im deutschsprachigen Raum darstellt.

\section{Häufige Indikationen}

Eine den häufigen Indikationen für Phytotherapeutika gewidmete Session thematisierte primär die Atemwegserkrankung und die Depression. Prof. D. Jobst zeigte auf, wie Phytopharmaka aus Efeu, Eibisch oder Wollblume in die Behandlung von Erkältungskrankheiten gut integriert sind, sei es als Fertigarzneimittel oder als Tee. Dr. S. Klement stellte die Ergebnisse einer randomisierten, doppelblinden und placebokontrollierten Studie vor, welche die Wirksamkeit eines Arzneimittels mit Lavendelöl (Silexan) bei Angst und depressiver Störung aufzeigte. Dr. R. Stange referierte über die Sicherheit und Compliance des Kombinationspräparates Contramutan N Saft in der Behandlung von akuten Atemwegsinfektionen und legte dar, dass das Komplexpräparat bei Kindern und Erwachsenen sicher erscheint und basierend auf den Sekundäranalysen eine gute Wirksamkeit aufweist. Prof. K. Kraft berichtete von positiven Erfahrungen mit der Phytotherapie und anderen komplementären Verfahren bei der Therapie infektanfälliger Kinder im Zusammenhang mit MutterKind-Kuren. Ergebnisse zur Wirkungsweise von Inhaltsstoffen des Griechischen Bergtees (Sideritis scardica) wurden durch einen jungen Wissenschaftler F. Heiner - vorgestellt. Er konnte am Modellorganismus Caenorhabditis elegans darstellen, dass Sideritis-scardica-Extrak- 
te hier eine neuroprotektive Wirkung aufweisen.

\section{Novel Targets}

Eine weitere Session beschäftigte sich mit Novel Targets. Die Untersuchung der Wirkungsweise von Phytopharmaka mit ihren pleiotropen Signaturen führt insbesondere beim Einsatz der „Omic“-Technologien immer wieder zur Entdeckung neuer, interessanter Targets, aber auch zur Aufklärung bisher unbekannter Pathomechanismen - wie in gleich zwei Vorträgen für den Kombinationsextrakt STW5 gezeigt wurde. Dr. H. Abdel-Aziz schlug den Orphanrezeptor GPR84 als neues Behandlungsziel für die Refluxösophagitis vor und Prof. K. Nieber berichtete über die Aktivierung von A2A-Rezeptoren als neuem, möglicherweise therapeutisch relevanten Wirkmechanismus zur Vermeidung morphologischer Schäden sowie zur Wiederherstellung der gestörten Motilität bei entzündlichen Darmerkrankungen.

Herr Dr. Scheffler referierte über die erfolgreiche Entwicklung und Zulassung einer Betulinemulsion auf der Basis eines triterpenreichen Trockenextraktes aus Birkenrinde zur Behandlung von Hautund Verbrennungswunden, wofür er im weiteren Verlauf des Kongresses mit dem

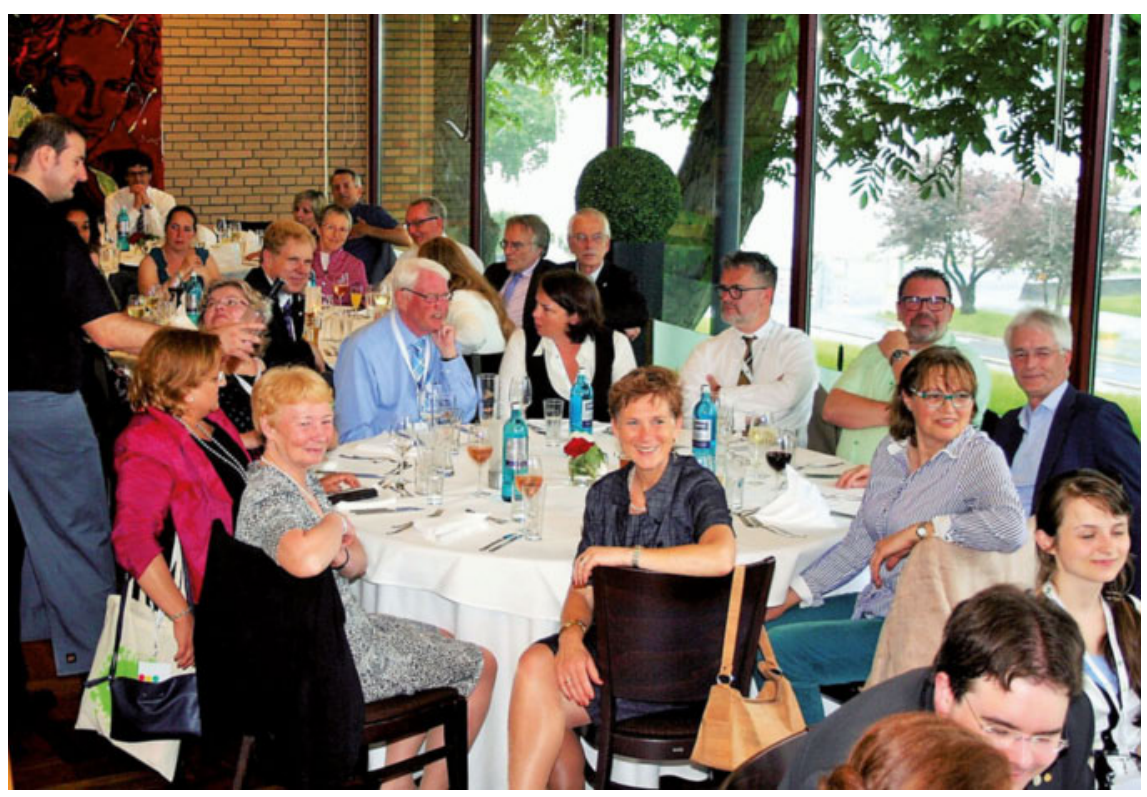

Der Gesellschaftsabend im „DaCapo“.
Innovationspreis der GPT ausgezeichnet wurde. S. Sarshar berichtete von ihren Arbeiten zu zytoprotektiven und antiadhäsiven Effekten eines wässrigen Extraktes aus Orthosiphon-Blättern gegen uropathogene E. coli und abschließend fasste Dr. J. Müller in einer Metaanalyse Aussagen zur Wirksamkeit und Studienqualität von STW 5 zusammen.

\section{Extraktentwicklung}

Wie schon programmatisch im Motto des diesjährigen Kongresses verankert, war eine Session der Extraktentwicklung gewidmet. Hier wurden die neuesten Megen in der Extraktentwicklung und Standardisierung pflanzlicher Extrakte diskutiert. Dr. B. Steinhoff thematisierte die Reinheitsanforderungen bzgl. Pestizid-, Schwermetall- und Aflatoxinrückständen, aber ging auch auf die aktuelle Pyrrolizidinalkaloidproblematik ein. Dr. H. Kurth stellte die Vorteile einer HPLC-Methode zur Vereinfachung der Gehaltsbestimmung von Hydroxyanthracen-Glykosiden aus Sennes-Früchten und -Blättern vor, die als bewährte Wirkstoffe für Laxantien eingesetzt werden. Vergleichende Untersuchungen zum Extraktionsverhalten der Anthranoide unter Berücksichtigung der Aglyka bei unterthoden, genauso wie die Herausforderun-

schiedlichen Extraktionsmitteln aus Sennesfrüchten, vorgestellt von Prof. B. Meier, schlossen sich an. Die Modernisierung eines traditionellen Arzneidrogengemisches aus der Kampo-Medizin im Sinne eines Traditional Herbal Medicinal Products wurde von Prof. K. Kuchta vorgestellt. Den Abschluss der spannenden und sehr praxisbezogenen Session bildetet der Beitrag von Frau Gorgus mit ihren vergleichenden Untersuchungen zum Alkoholgehalt in Lebensmitteln und Phytotherapeutika und ihrer Bewertung im Rahmen der Kinderheilkunde.

\section{Der Gesellschaftsabend}

Ein Highlight des Kongresses war zweifellos der Gesellschaftsabend. Sowohl die unterhaltsamen Jazz-Darbietungen der musizierenden Vorstandsmitglieder Dr. Tankred Wegner und Dr. Bruno Frank als auch das kulinarisch verwöhnende Angebot des Restaurants DaCapo, das während des gesamten Kongresses für das leibliche Wohl der Teilnehmer sorgte, trugen maßgeblich zu einem rundum gelungenen Gesellschaftsabend bei. Prof. Michael Keusgen, der in seiner spritzigen und „nicht ganz bierernsten“ Festrede die Vorzüge eines - leider vorerst noch imaginären - cannabinoidhaltigen Bieres als einem definitiven Blockbuster der Phytotherapie der Zukunft anpries, förderte die gute Stimmung weiter. GPTPräsidentin Prof. Karin Kraft und Kongresspräsidentin PD Dr. Ulrich-Merzenich dankten allen, die durch ihr - größtenteils ehrenamtliches - Engagement zum guten Gelingen des Phytotherapie-Kongresses 2016 beigetragen haben.

\section{Von Cimicifuga bis Weißdorn}

Am Samstag setzte sich das vielseitige und anspruchsvolle Kongressprogramm fort. Dr. H.-H. Henneicke-von Zepelin präsentierte die 60-jährige Entwicklungsgeschichte der Arzneipflanze Cimicifuga zu einem „Phyto-Blockbuster“ in der Therapie der Wechseljahrsbeschwerden, gekennzeichnet von intensiven und kontinuierlichen Forschungsarbeiten. Dr. A. Schapowal stellte aktuelle Ergebnisse zu 


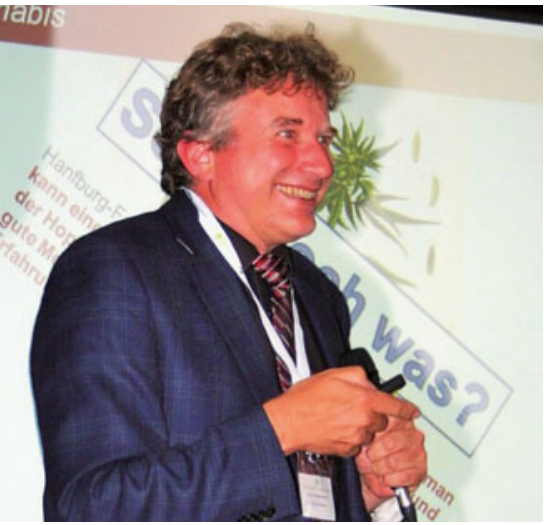

Alles andere als bierernst: Prof. Michael Keusgen bei der Festrede.

Echinacea-Präparaten vor, die in einer Metaanalyse zusammengefasst nahe legen, dass die Therapie von Atemwegserkrankungen mit pflanzlichen Arzneimitteln aus Echinacea das Risiko rezidivierender Infekte gegenüber Placebo signifikant vermindern, wobei sich lipophile Extrakte als signifikant wirksamer erwiesen als Presssaftzubereitungen. Prof. B. Meier räumte anschließend in seinem lebendigen Beitrag „Heißes Wasser als Extraktionsmittel“" mit dem Mythos auf, dass Wasser für arzneiliche Zubereitungen kein geeignetes Extraktionsmittel sei. Auch das Europäische Arzneibuch enthält eine Reihe von Extraktmonografien, für die Wasser als Auszugsmittel empfohlen wird. Die Beispiele reichten von Trockenextrakten aus Melissen- oder Pfefferminzblättern, über Baldrianwurzeln hin zu eigenen Analysen der C-Glykoside aus Weißdorn und einfachen Glykosiden wie dem Arbutin und dem Salicin. Wasser erweist sich als vielfältiges und unverzichtbares Extraktionsmittel, wobei für die industrielle Extraktproduktion Ethanol/ Wasserextraktionen in der Regel vorteilhafter sind. Zum Abschluss der Session stellte Dr. H. Sievers eine Bestandsaufnahme der regulatorischen HMPC-Dokumente zur toxikologischen Bewertung von pflanzlichen Arzneimitteln vor und präsentierte Beispiele einiger Grenzwertfestlegungen. Sievers und Co-Worker kamen zum Schluss, dass hier Dokumente bereitstehen, die eine gemeinsame Orientierung für Antragsteller und Behörden darstellen und Zulassungsverfahren erleichtern können.

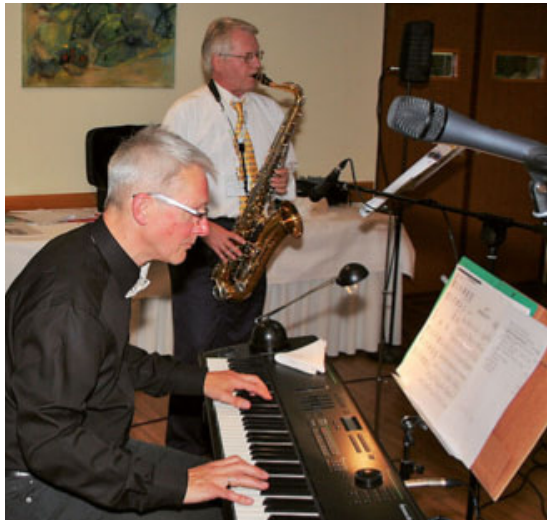

Die GPT jazzt: Dr. Tankred Wegener und Dr. Bruno Frank.

\section{Traditionelle Wurzeln und Zukunft}

Die Abschluss-Session war der Tradition und - insbesondere - der Zukunft der Phytotherapie sowie den Preisverleihungen gewidmet. Dr. J.G. Mayer stellte zunächst die Arbeit der Forschergruppe Klostermedizin aus Würzburg vor. Sie reicht von der Erstellung von Datenbanken zur Dokumentation der im Mittelalter in Europa arzneilich verwendeten Pflanzen über aktuelle Recherchen bis hin zur direkten Wissensweitergabe in Form von Universitätsvorlesungen, Vorträgen und Workshops für Laien und einer intensiven Medienarbeit, mit der rund 250000 Personen pro Monat erreicht werden. Prof. $H$. Stuppner legte anschließend überzeugend die großen $\mathrm{Zu}$ kunftspotenziale der Phytotherapie aus pharmazeutischer Sicht dar. Er machte wie schon Prof. M. Keusgen - deutlich, dass bisher von den ca. 300000 weltweit vorkommenden Pflanzenarten nur etwa 5-10\% chemisch bzw. pharmakologisch ausreichend gut untersucht sind und damit der riesige pflanzliche Arzneimittelschatz immer noch weitgehend unbearbeitet ist. Phytopharmaka als Vielstoffgemische stellen bezüglich ihrer Qualitätssicherung und der Erforschung ihrer Wirkmechanismen zwar eine Herausforderung dar, aber ganzheitliche Ansätze und die Systembiologie bieten neue und vielversprechende Instrumente zur Beschreibung und zum Verständnis derartiger komplexer Multiparameter-Systeme an. Die Phytotherapie bleibt damit ein

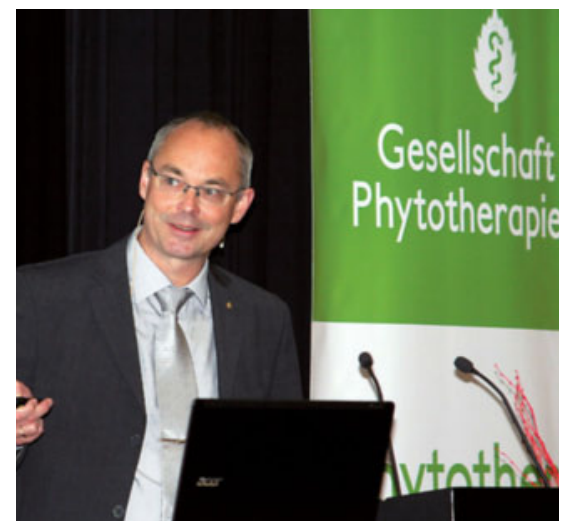

Dr. H.-H. Henneicke-von Zepelin

spannendes und zukunftsfähiges Forschungsgebiet! Prof. R. Saller stellte abschließend aus medizinischer Sicht die genuinen pleiotropen Multitarget-Eigenschaften der Phytotherapeutika heraus, die es erlauben, eine „Polypharmakologie mit einem einzigen Wirkstoff“ zu betreiben. Wegen ihres breiten Wirkungsspektrums könnten sie sich auch in komplexen Therapiesituationen als geeignete Therapiebestandteile erweisen, gewissermaßen als polypharmakotherapeutische Ansätze. Er schlug vor, dass neben der rein stofflichen Betrachtungsweise auch funktionell orientierte Herangehensweisen unmittelbar für eine Therapieentscheidung relevant sein können.

\section{Preise}

Der mit 10000 Euro dotierte Innovationspreis der Gesellschaft für Phytotherapie wurde an Herrn Dr. Armin Scheffler (Birken AG) verliehen. Die GPT honorierte damit die Entwicklung und Zulassung eines Arzneimittels aus dem triterpenreichen Trockenextrakt der Birkenrinde, welches in Form einer Betulin-Emulsion zur Behandlung von oberflächlichen Hautwunden und Verbrennungswunden der Haut vom Grad Ila bei Erwachsenen angewendet wird (s. ZPT 3, S. 125).

Mit Posterpreisen wurden Frau S. Sarshar (Pharmazie Münster), Frau C. Vissiennon (Pharmazie Leipzig) und Frau K. Braun (Züricher Hochschule für angewandte Wissenschaften) ausgezeichnet. S. Sarshar stellte sehr beeindruckend Invivo- und In-vitro-Daten zur Hemmung 


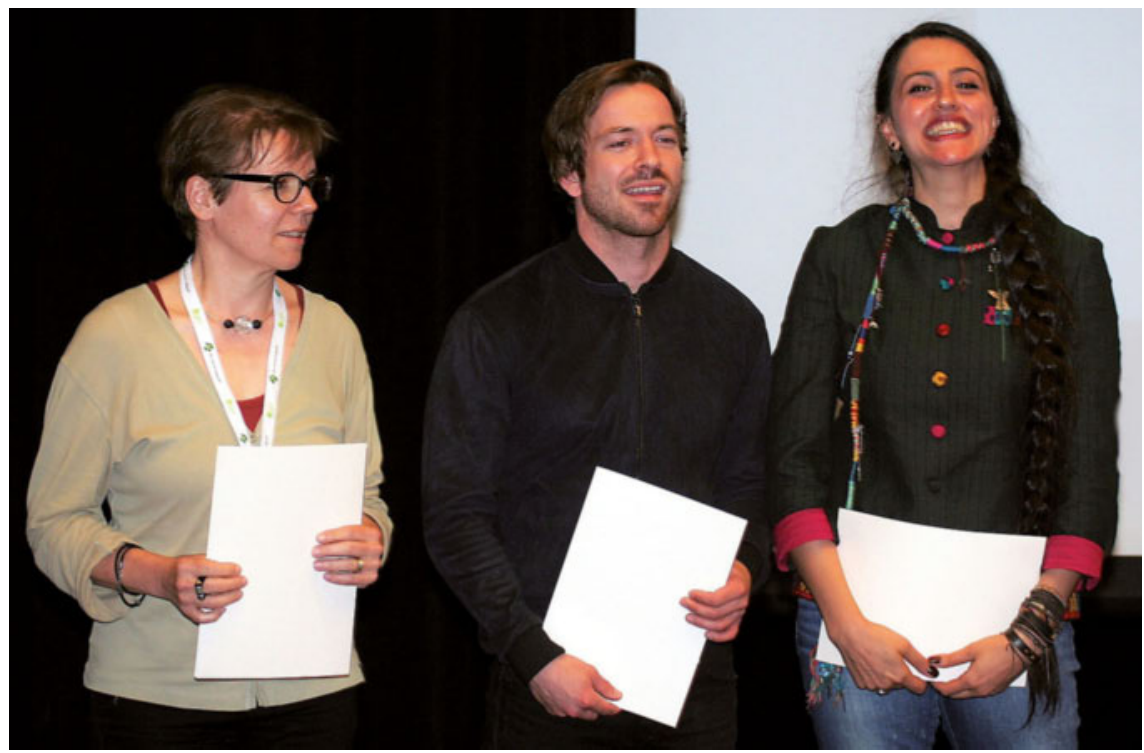

Der Mühen Lohn: Die Posterpreise gingen an Dr. Beatrix Falch (stellvertretend für die Arbeitsgruppe K. Braun et al.), Peter Marquardt (stellvertretend für Dr. Cica Visiennon) und Shabnam Sarshar.

der Adhäsion von uropathogenen Keimen durch einen wässrigen Extrakt aus Blättern von Orthosiphon stamineus vor. Dr. C. Vissiennon überzeugte die Jury mit ihrem Kombinationsscreening von Kamille, Myrrhe und Kaffeekohle in Bezug auf die Chemokinfreisetzung aktivierter humaner Makrophagen. K. Braun wurde für ihre Untersuchungen zum Extraktionsverhalten der Anthranoide in Sennae folium/fructus unter Berücksichtigung der Aglyka geehrt.

Am Ende des Kongresses zog Kongresspräsidentin PD Dr. G. Ulrich-Merzenich ein sehr positives Fazit und dankte allen Referenten für ihre Beiträge sowie den Teilnehmern für ihre aktive Mitwirkung

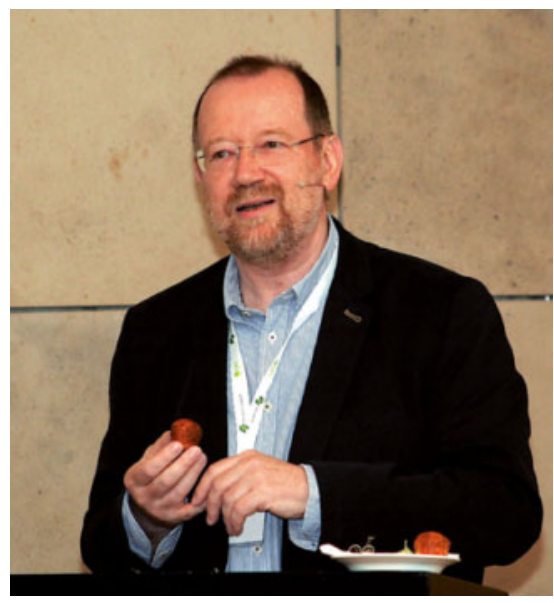

Kulinarische Archäologie: Prof. Dr. Josef Matzerath. zum guten Gelingen des vielseitigen und anspruchsvollen wissenschaftlichen Programms. Sie lud im Namen der GPT zum nächsten Phytotherapie-Kongress ein, der vom 28.-30. September 2017 in Münster stattfinden wird.

Zum Abschluss setzten Prof. J. Matzerath und Gourmetkoch V. Nebrich noch einmal ein Highlight mit ihrer „kulinarischen Archäologie“, in der sie auf hohem kulinarischen und unterhaltsamen akademischem Niveau die von ihnen wiederbelebte historische Küche des sächsischen Hofes in Dresden als moderne, gesunde und sinnliche Produktküche vorstellten.

\section{Phytotherapeutische Tiermedizin}

Im Rahmen des Kongressprogramms fand zusätzlich am Samstag ein kurzes, aber spannendes und gut besuchtes Symposium für Tiermediziner statt einschließlich einer speziellen Postersession. Vorträge und rege Diskussionen beschäftigten sich mit der Misteltherapie bei Tumorerkrankungen von Hund, Katze und Pferd, mit der Behandlung von Wundinfektionen mit multiresistenten Erregern durch Phytotherapeutika, mit der Therapieoptimierung der Antibiose durch Phytotherapeutika oder mit der anthelmintischen Wirkung von Pflanzen. Auch Unwetterwarnungen konnten zahlreiche hoch motivierte Kongressteilnehmer am Samstag nach fast drei Tagen Kongressprogramm nicht davon abhalten, noch an Führungen durch den Botanischen Garten am Schloss Poppelsdorf teilzunehmen. Diese wurden mit Begeisterung angenommen und machten Lust auf ein Wiederkommen nach Bonn.

Der Phytotherapie-Kongress 2016 war der bisher am besten besuchte Kongress der GPT in Deutschland und - nicht zuletzt dank der aktiven Beteiligung der schweizerischen und österreichischen Partner - derjenige mit dem umfangreichsten wissenschaftlichen Programm. Von den Teilnehmern wurde der gelungene „Dreiklang“ aus wissenschaftliche Beiträgen, begeisternder Musik und kulinarischen Genüssen sehr gelobt, der zusammen mit der guten Kongressorganisation, den angenehmen Räumlichkeiten und der reibungslosen Konferenztechnik während des gesamten Kongresses zu einer sehr guten und entspannten Atmosphäre beitrug. Das zeitweise tropische Wetter mit monsunartigen Regengüssen tat der guten Stimmung keinen Abbruch.

\section{Fazit}

Die Phytotherapie wird als Methode in der Medizin stärker wahrgenommen als früher, aber sie kann und muss noch deutlich „gewichtiger“ werden. Die uns heute zugänglichen Forschungsmethoden im Bereich der Grundlagenforschung sind geeignet, dies im Sinne einer evidenzbasierten Medizin zu bewerkstelligen. Nun gilt es, mehr junge Wissenschaftler und Ärzte zu gewinnen, die aktiv Grundlagenforschung betreiben und die gewonnenen Erkenntnisse in ganzheitliche Therapiekonzepte umsetzen.

\section{Gudrun Ulrich-Merzenich/Bernd Merzenich}

Fotos: Lothar Geisler

Die Kongressbeiträge können im Abstraktband (Zeitschrift für Phytotherapie, Supplement 1, 2016) oder online nachgelesen werden (https://www. thieme-connect.de/products/ejournals/issue/ 10.1055/s-006-31837). 